\title{
SPECIFIC HEAT OF Cr-BASED SEMIMAGNETIC SEMICONDUCTORS
}

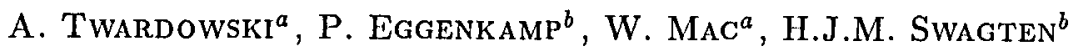 \\ AND M. DEMIANIUK ${ }^{c}$ \\ ${ }^{a}$ Institute of Experimental Physics, Warsaw University \\ Hoża 69, 00-681 Warszawa, Poland \\ ${ }^{b}$ Physics Department, Eindhoven University of Technology \\ PO Box 513, 5600 MB Eindhoven, The Netherlands \\ c Institute of Technical Physics, WAT, Warszawa, Poland
}

\begin{abstract}
Specific heat of $\mathrm{ZnCrSe}$ and $\mathrm{ZnCrS}$ was measured for $1.5<T<10 \mathrm{~K}$ and $B<3 \mathrm{~T}$. The data were interpreted using "crystal field model" taking into account tetrahedral crystal field, tetragonal Jahn-Teller distortion and spin-orbit interaction.
\end{abstract}

PACS numbers: 75.50.Pp, 71.70.Ch, 71.70.Ej

Recently a new class of semimagnetic semiconductors (SMSC) with $\mathrm{Cr}$ as magnetic ion was synthesized [1-3]. From magnetic point of view Cr-SMSC represents the case of an intermediate magnetic material: between classical Brillouin-type paramagnets (like Mn and Co-SMSC) and Van Vleck-type systems (Fe-SMSC) [4]. This particular magnetic behaviour results from the static JahnTeller effect suffered by $\mathrm{Cr}$ ions, leading to a doublet ground state, followed by a closely lying (about $10 \mathrm{~K}$ ) doublet and a singlet $[5,6]$.

In order to check this energy structure we studied low-temperature specific heat of $\mathrm{Zn}_{1-x} \mathrm{Cr}_{x} \mathrm{~S}(x=0.0065)$ and $\mathrm{Zn}_{1-x} \mathrm{Cr}_{x} \mathrm{Se}(x=0.004)$. We used standard heat pulse method in the temperature range $1.5<T<10 \mathrm{~K}$ and magnetic field up to $2.8 \mathrm{~T}$. Magnetic contribution to the specific heat was obtained by subtracting pure host lattice specific heat from the total heat capacity of the sample.

In the absence of magnetic field specific heat shows for both materials a Shottky-type anomaly (Figs. 1 and 2) with the energy gap of the order of $10 \mathrm{~K}$ ( $10 \mathrm{~K}$ for $\mathrm{ZnCrSe}$ and $7.7 \mathrm{~K}$ for $\mathrm{ZnCrS}$ ). In the presence of magnetic field specific heat strongly increases which is ascribed to the splitting of the ground state doublet.

We describe our data using simple "crystal field" model taking into account tetrahedral crystal field, static tetragonal Jahn-Teller distortion and spin-orbit interaction [5, 1]. The parameters for the model are provided by independent experiments (FIR and IR spectroscopy) [5,6]. The details of calculations are described elsewhere [1]. We find reasonable overall agreement between experimental 


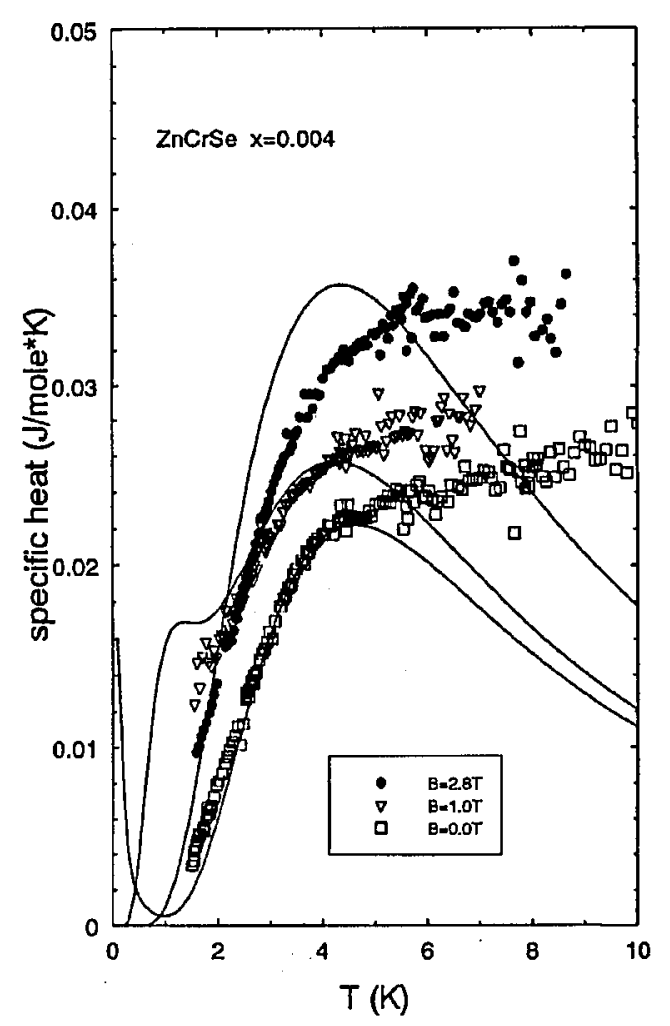

Fig. 1. Magnetic specific heat of $\mathrm{Zn}_{1-x} \mathrm{Cr}_{x} \mathrm{Se}(x=0.004)$ as a function of temperature at $B=0,1$ and $2.8 \mathrm{~T}$. The lines represent calculations as described in the text for $x=0.0045$ and $D q=-500 \mathrm{~cm}^{-1}, B_{2}^{0}=5 B_{4}^{0}=-85.71 \mathrm{~cm}^{-1}$, and $\lambda=86 \mathrm{~cm}^{-1}$ $(\boldsymbol{B} \|(111))$.

data and the model (Figs. 1 and 2). We notice that in-field results should be considered with care, since they were calculated for (111) cubic direction which is not real experimental situation. For $\mathrm{ZnS} \mathrm{X}$-ray analysis indicated that symmetry of "(111)" direction is slightly different from typical cubic (111) symmetry, which is ascribed to politype structure of our crystals. On the other hand, $\mathrm{ZnCrSe}$ sample was not really oriented in respect to the field direction since no single crystals of suitable size were available. Anyway in both cases in-field behaviour of specific heat is quite well recovered.

There is however some deviation between experiment and theory in the absence of magnetic field at the lowest temperatures $(T<4 \mathrm{~K})$ as well as for the high-temperature part. Both of them are not currently understood and it points to existence of additional energy states not taken into account by our model. In particular, low-temperature data suggest existence of additional energy gap, smaller than predicted for isolated $\mathrm{Cr}$ ion (estimated to be about $6 \mathrm{~K}$ for $\mathrm{ZnCrS}$ and $8 \mathrm{~K}$ for $\mathrm{ZnCrSe}$ ). This could result from the possible exchange $d-d$ interaction between 


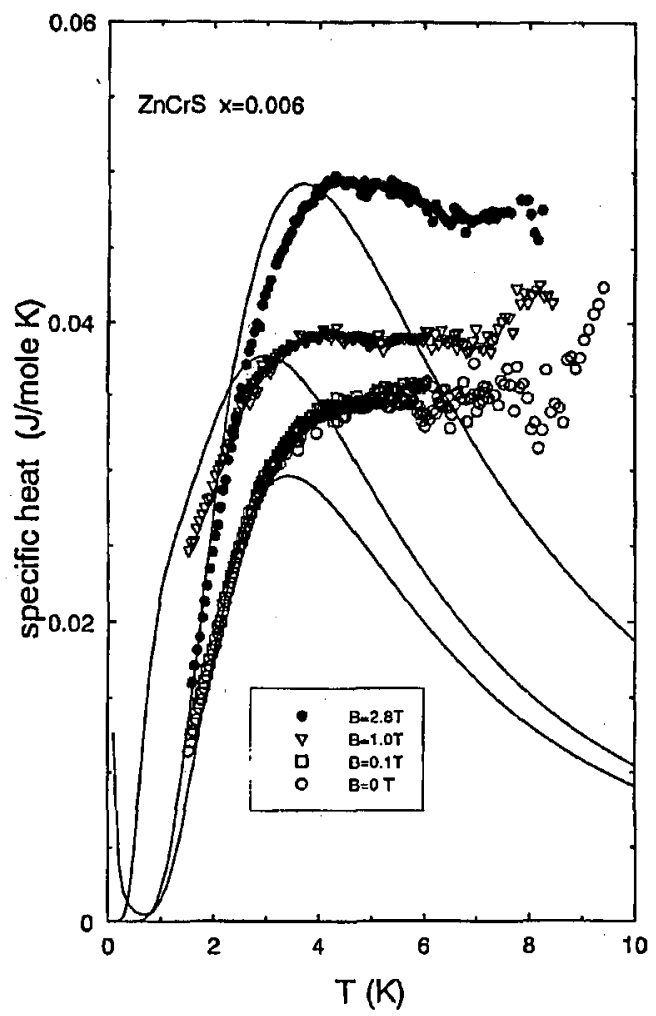

Fig. 2. Magnetic specific heat of $\mathrm{Zn}_{1-x} \mathrm{Cr}_{x} \mathrm{~S}(x=0.0065)$ as a function of temperature at $B=0,0.1,1$ and $2.8 \mathrm{~T}$. The lines represent calculations as described in the text for $x=0.006$ and $D q=-520 \mathrm{~cm}^{-1}, B_{2}^{0}=5 B_{4}^{0}=-89 \mathrm{~cm}^{-1}$ and $\lambda=77 \mathrm{~cm}^{-1}(B \|(111))$.

Cr ions or ligand effects not taken into account in our model. This problem requires further study, for which crystals with higher $\mathrm{Cr}$ concentration would be particularly useful.

\section{References}

[1] A. Twardowski, T. Fries, Y. Shapira, P. Eggenkamp, H.J.M. Swagten, M. Demianiuk, J. Appl. Phys. 73, 10 (1993).

[2] W. Mac, Nguyen The Khoi, A. Twardowski, J.A. Gaj, M. Demianiuk, Acta Phys. Pol. A 82, 902 (1992).

[3] R. Krevet, A. Twardowski, M. von Ortenberg, W. Mac, M. Demianiuk, Solid State Commun., in press.

[4] Diluted Magnetic, in series Semiconductors Semiconductors and Semimetals, Vol. 25, Eds. J.K. Furdyna, J. Kossut, Academic Press, San Diego 1988; Diluted Magnetic Semiconductors, Eds. M. Balkanski, M. Averous, Plenum Press, New York 1991; J.K. Furdyna, J. Appl. Phys. 64, R29 (1988).

[5] J.T. Vallin, G.A. Slack, S. Roberts, A.E. IIughes, Phys. Rev. B 2, 4313 (1970).

[6] J.T. Vallin, G.D. Watkins, Phys. Rev. B 9, 2051 (1974). 